\title{
Association Analysis of Persistent Cytopenia With Efficacy and Side Effects of Anti-CD19 CAR T Therapy in Relapsed/refractory Diffuse Large B-Cell Lymphoma Patients.
}

\section{Xuemei Lin}

Tianjin First Central Hospital https://orcid.org/0000-0002-1492-0057

\section{Meijing Liu}

Tianjin First Central Hospital

\section{Rui Cui}

Tianjin First Central Hospital

\section{Cuicui Lyu}

Tianjin First Central Hospital

\section{Jia Wang}

Tianjin First Central Hospital

\section{Qing Li}

Tianjin First Central Hospital

\section{Nan Mou}

Shanghai Genbase Biotechnology

Qi Deng ( $\square$ kachydeng@126.com )

Tianjin First Central Hospital https://orcid.org/0000-0002-3646-4953

\section{Research Article}

Keywords: Chimeric antigen receptor (CAR), Diffuse large B-cell lymphoma, Cytopenia, Efficacy, Side effects

Posted Date: January 18th, 2022

DOI: https://doi.org/10.21203/rs.3.rs-1254282/v1

License: (9) (1) This work is licensed under a Creative Commons Attribution 4.0 International License. Read Full License 


\section{Abstract}

Background: Anti-CD19 chimeric antigen receptor (CAR) T cell therapy has been an effective salvage therapy for relapsed/refractory (R/R) diffuse large B-cell lymphoma (DLBCL). Hematological toxicity is one of the adverse events (AEs) of this therapy. But the underlying pathophysiologic mechanism of persistent cytopenia is unclear. The association of persistent cytopenia with the efficacy and side effects of anti-CD19-CAR T cell therapy should be concerned about.

Patients and methods: In this work, 38 R/R DLBCL patients were enrolled in clinical trial of anti-CD19-CAR T-cell therapy. Before CAR T-cell therapy, all the patients received lymphodepleting chemotherapy with fludarabine and cyclophosphamide. The degree and duration of cytopenia, clinical response, proportions of CAR T-cells and levels of interleukin-6 (IL-6), AEs and follow up were observed following the anti-CD19CAR T-cell therapy.

Results: The 3-4 grade of persistent cytopenia occurred in $14 \mathrm{R} / \mathrm{R}$ DLBCL patients (more than 8 weeks after CAR-T cell infusion) and recovered at 8-18 weeks after CAR-T cell infusion. All these 14 patients achieved objective response rate (ORR) in their anti-CD19-CAR T cell therapy. In the 26 patients obtained ORR, the incidence of 3-4 grade of persistent cytopenia was higher in patients with high tumor load than that of patients without high tumor load. The mean peak of IL-6 and anti-CD19-CAR T cells, the grade of CRS in patients with 3-4 grade of persistent cytopenia were higher than that of patients without such persistent cytopenia. Most patients whose anti-CD19-CAR T cells could be observed at 21 and 28 days after infusion were associated with 3-4 grade of persistent cytopenia. The PFS and OS were higher in patients with 3-4 grade of persistent cytopenia.

Conclusion: All the R/R DLBCL patients who occurred 3-4 grade of persistent cytopenia after anti-CD19CAR T cell therapy achieved ORR in this therapy. The 3-4 grade of persistent cytopenia was associated with high tumor load, higher peaks of IL-6 and anti-CD19-CAR T cells, higher grades of CRS, the higher PFS and OS. Our study is helpful in predicting efficacy and severe hematologic side effects of anti-CD19CAR T-cell therapy in R/R DLBCL patients.

Trial registration: The study was registered at http://www.chictr.org.cn/index.aspx as ChiCTR-ONN16009862 and http://www.chictr.org.cn/index.aspx as ChiCTR1800019622.

\section{Introduction}

Diffuse large B-cell lymphoma (DLBCL) is a common type of B-cell non-Hodgkin lymphoma (B-NHL). Although combination chemotherapy based on R-CHOP (Rituximab, Cyclophosphamide, Doxorubicin, Vincristine, and Prednisone) achieved a higher objective response rate (ORR) in DLBCL, several patients developed to relapsed/refractory (R/R) DLBCL after standard combined chemotherapy [1-3]. Anti-CD19 chimeric antigen receptor (CAR) T cell therapy has been an effective salvage therapy for R/R DLBCL [47]. But its utility is hampered by the adverse events (AEs). These AEs include cytokine release syndrome (CRS), immune effector cell associated neurotoxic syndrome (ICANS), hematological toxicity, B-cell 
aplasia, and so on [8-14]. Hematologic toxicity is a factor associated with secondary infection after antiCD19-CAR T-cell therapy. In long-term activity and safety outcomes of the ZUMA-1 study, there was less than $20 \%$ of patients had grade 3 or worse cytopenias three months after this therapy [4]. But the underlying pathophysiologic mechanism of prolonged cytopenias is unclear. Fortunately, none of these patients had serious clinical sequelae due to this persistent cytopenia $[15,16]$. Could this persistent cytopenia be explained by lymphodepleting chemotherapy with fludarabine and cyclophosphamide alone? Some studies before showed that the risk factors associated with persistent cytopenia include CRS/ICANS grade, baseline cytopenia, prior allogeneic stem cell transplantation, higher peak C-reactive protein or ferritin levels $[17,18]$. The association of persistent cytopenia with the efficacy and side effects of anti-CD19-CAR T cell therapy should be of clinical concern.

\section{Patients And Methods}

\section{Medical history of the patients enrolled in this study}

Thirty-eight R/R DLBCL patients were admitted in our hospital and enrolled in clinical trials of anti-CD19CAR T-cell therapy (ChiCTR-ONN-16009862 and ChiCTR1800018059) from January 2017 to May 2021. All these patients were diagnosed as R/R DLBCL. None of the patients received hematopoietic stem cell transplant (HSCT) before and after our study. Follow-up was carried out from the CAR-T cell infusion day to the date of cutoff or the date of death. The cutoff date was November 30, 2021 in this study.

\section{Anti-CD19-CAR T cell therapy}

All 38 R/R DLBCL patients provided their autologous peripheral blood mononuclear cells (PBMCs) for their anti-CD19-CAR T-cell therapy. The lenti-CD19-2rd-CAR was provided by Shanghai Genbase Biotechnology Co., Ltd. Shanghai, CHINA. After 12-15 days cell culture in vitro, the transduction efficiency of the anti-CD19-CAR was analyzed by flow cytometry (FCM). Before anti-CD19-CAR T-cell therapy, all the patients received lymphodepleting chemotherapy with fludarabine $\left(30 \mathrm{mg} / \mathrm{m}^{2}\right)$ and cyclophosphamide $\left(400 \mathrm{mg} / \mathrm{m}^{2}\right)$ from day -4 to day -2 . Anti-CD19-CAR T cells were infused on day $0\left(2 \times 10^{6} \mathrm{cells} / \mathrm{kg}\right)$ in all R/R DLBCL patients.

\section{Clinical response criteria}

From the date of anti-CD19-CAR T cells infusion, follow-up was done until the patients died. Two months after the anti-CD19-CAR T cell therapy, the efficacy was evaluated in all the $38 \mathrm{R} / \mathrm{R}$ DLBCL patients. Disease status was defined as complete response (CR), partial remission (PR), stable disease (SD) and progression of disease (PD) according to Lugano Revised Criteria for Response Assessment [19]. In our study, we observed the progression free survival (PFS) and overall survival (OS) after the anti-CD19-CAR T-cell therapy.

\section{Adverse events (AEs) of anti-CD19-CAR T-cell therapy}


The AEs were observed more than 120 days following the anti-CD19-CAR T-cell infusion. Cytokine release syndrome (CRS) grade was determined according to the National Cancer Institute Common Terminology Criteria for AE v4.03 [20]. And the neurotoxicity syndrome was determined according to the Immune effector cell associated neurotoxic syndrome (ICANS) [21].

\section{The expansion of anti-CD19-CAR T cells and levels of cytokines}

The proportions anti-CD19-CAR T cells in peripheral blood were examined by FCM on 0, 7, 14, 21, 28 and 60 days after CAR-T cell infusion. Levels of interleukin-6 (IL-6) was measured on day 0, 7, 14, 21 and 28 after CAR T-cell infusion by enzyme linked immunosorbent assay (ELISA).

\section{Criteria of CAR T-cell therapy mediated hematotoxicity and persistent cytopenia}

The criteria of CAR T-cell therapy mediated hematotoxicity was graded according to the joint ASCO/IDSA consensus guidelines for cancer-related infection risk [22,23]. In our study, the persistent cytopenia was defined as 3-4 grade of cytopenia and hematotoxicity lasting more than 56 days after CAR-T cell infusion (Because in most patients, AEs in addition to hematotoxicity disappeared at 21-28 days after CAR-T cell infusion).

\section{Statistical analysis}

SPSS 17.0 (SPSS Inc., Chicago, IL, USA) software was used for all the statistical analyses. T test was used for the measurement data conforming to normal distribution, and rank sum test was used for the measurement data not conforming to normal distribution. Chi-square test was used for counting data. All values were expressed as mean $\pm \mathrm{SD}$. $P<0.05$ was considered statistically significant.

\section{Results}

\section{Patient characteristics}

Baseline characteristics of the 38 R/R DLBCL patients enrolled in this clinical trial were listed in Table 1. The age, the molecular subtypes, the stages according to the modified Ann Arbor staging system, the international prognostic index (IPI) scores, adverse prognostic factors of double hit, triple hit or double expression, triple expression and the lines of therapies were listed. None of the patients had any level of cytopenia when they were enrolled. In addition to the mean max tumor diameter, there was no difference in characteristics between the $14 \mathrm{R} / \mathrm{R}$ DLBCL patients with 3-4 grade of persistent cytopenia and the 24 R/R DLBCL patients without such persistent cytopenia after their anti-CD19-CAR T-cell therapy (Table 2).

\section{Transduction efficiency, amplification and infusion of the anti-CD19-CAR T cells}

The mean CD19-CAR transduction efficiency in the final products of the $38 \mathrm{R} / \mathrm{R}$ DLBCL patients was $49.85 \pm 13.35 \%$. All the 38 patients received a dose of $2.19 \pm 0.42 \times 10^{6}$ cells $/ \mathrm{kg}$ anti-CD19-CAR T cells infusion on day 0 after lymphodepleting chemotherapy. 


\section{Hematological toxicity of anti-CD19-CAR T-cell therapy}

The hematological toxicity was diagnosed with 0-4 grade after anti-CD19-CAR T-cell therapy. It occurred from 4 to 10 days post anti-CD19-CAR T-cell infusion. 13 patients were defined as $3-4$ grade of neutropenia, 9 patients were defined as 3-4 grade of anemia and 11 patients were defined as 3-4 grade of thrombocytopenia in this study (Figure 1a). The 3-4 grade of persistent cytopenia occurred in 14 patients in all these $38 \mathrm{R} / \mathrm{R}$ DLBCL patients (the hematotoxicity lasting more than 8 weeks after CAR-T cell infusion). The different types and persistent time of cytopenia in all these 14 patients who were defined as 3-4 grade of cytopenia are shown in Figure 1b. The trend of blood cell changes in patients with 3-4 grade of neutropenia, anemia and thrombocytopenia is shown in Figure $1 \mathrm{c} \mathrm{d}$ e. We could see that the trend curve of grade 3-4 neutropenia has two valleys in seven patients with persistent neutropenia (Pt 2, 4, $14,16,21,32,34)$. Mean value of the first valley of neutropenia was $0.66 \pm 0.21 \times 10^{9} / \mathrm{L}$ at 7 days after CAR-T infusion, while the second valley of neutropenia was $0.21 \pm 0.11 \times 10^{9} / \mathrm{L}$ at $28-56$ days after CAR-T infusion (Figure 1c). Such change trend was also observed in three patients (Pt 17, 21, 34) with persistent thrombocytopenia (Figure 1d). But this change trend was not observed in patients with persistent anemia.

There is no evidence of severe marrow dysplasia or relapse by bone marrow smear 8 weeks after CAR-T cell infusion in all these14 patients with 3-4 grade of persistent cytopenia. All these 14 patients with 3-4 grade of persistent cytopenia received supportive therapy to recover their persistent cytopenia. It was recovered at 8-18 weeks after CAR-T cell infusion.

After anti-CD19-CAR T-cell infusion, 3 patients who were diagnosed with 3 and 4 grade of neutropenia and 1 patients who were diagnosed with 2 grade of neutropenia were diagnosed as bacterial infections after anti-CD19-CAR T-cell therapy. All of these infections occurred in the period of their 3-4 grade of neutropenia. None of them had been diagnosed with invasive fungal disease. Only one patient (Pt 10) died of bacterial septicemia when the disease progressed again, but her neutropenia had been recovered at that time.

\section{The clinical response of CAR-T cells}

Two months after the anti-CD19-CAR T cell infusion, the efficacy of all the 38 R/R DLBCL patients was evaluated. 18 patients $(18 / 38,47.37 \%)$ obtained complete response (CR), while 8 patients $(8 / 38,21.05 \%)$ obtained partial remission (PR) in their anti-CD19-CAR T cell therapy. The ORR was $68.42 \%(26 / 38)$ in our study. 6 patients $(6 / 38,15.79 \%)$ obtained stable disease (SD), while other 6 patients $(6 / 38,15.79 \%)$ obtained progression of disease (PD) in .

All the 14 patients with 3-4 grade of persistent cytopenia obtained CR and PR in our study, while no patient who obtained SD and PD had 3-4 grade of persistent cytopenia after their anti-CD19-CAR T-cell therapy (Figure 2a).

In all the 18 R/R DLBCL patients who achieved CR, 15 patients survived in CR state until the cutoff date, the other three patients died of disease relapse (Pt 7,25 with persistent cytopenia, Pt 4 without persistent 
cytopenia). Only three patients who achieved PR (Pt 22,26,35 with persistent cytopenia) and two patients who achieved SD (Pt 8,13 without persistent cytopenia) survived until the cutoff date. All patients who achieved PD died of disease progression again (Figure 2b).

In all the $26 \mathrm{R} / \mathrm{R}$ DLBCL patients who obtained ORR in their anti-CD19-CAR T cell therapy, 14 patients had high tumor load and 12 patients had no high tumor load. In these 26 patients, 3-4 grade of persistent cytopenia occurred in $11(11 / 14,78.57 \%)$ patients with high tumor load, while in three $(3 / 12,25.00 \%)$ patients without high tumor load. Incidence of 3-4 grade persistent cytopenia was higher in patients with high tumor load than that of in patients without high tumor load in these 26 patients obtained ORR $(P=0.006)$ (Figure 2c).

\section{AEs of anti-CD19-CAR T-cell therapy}

The AEs were observed more than 120 days following the anti-CD19-CAR T-cell infusion. The patients developed fever, headache, fatigue, nausea, anorexia, dyspnea, cough, tachycardia and other symptoms. In addition to hematologic toxicity, there was no difference in characteristics between the $14 \mathrm{R} / \mathrm{R}$ DLBCL patients with 3-4 grade of persistent cytopenia and the $24 \mathrm{R} / \mathrm{R}$ DLBCL patients without such persistent cytopenia after anti-CD19-CAR T-cell therapy (Table 3). In most patients, AEs in addition to hematological toxicity disappeared after 14-21 days of CAR-T cell infusion.

The mean peak of IL- 6 in R/R DLBCL patients with 3-4 grade of persistent cytopenia (54.44 \pm 28.96 $\mathrm{pg} / \mathrm{mL})$ was higher than that of in patients without such persistent cytopenia $(17.06 \pm 8.22 \mathrm{pg} / \mathrm{mL})(P]$ 0.0001) (Figure 3a).

In addition to five patients developed 3 grade of CRS, all the other patients developed 0-2 grade of CRS in their anti-CD19-CAR T-cell therapy. Two patients developed 2 grade of ICANS, seven patients developed 1 grade of ICANS, then the other patients developed 0 grade of ICANS in this anti-CD19-CAR T-cell therapy. The grades of CRS were higher in patients with 3-4 grade of persistent cytopenia than that of patients without such persistent cytopenia $\left(P_{C R S}{ }^{\boxplus 0.0001}\right)$. But we did not observe this result in patients with different grades of ICANS ( $P_{\text {ICANS }}=0.0740$ ) (Figure $3 \mathrm{bc}$ ). None of the patients died of any level of CRS or ICANS during their anti-CD19-CAR T-cell therapy. Only patients who developed 3 grade of CRS and 2 grade of ICANS received glucocorticoid or tocilizumab in anti-CD19-CAR T-cell therapy.

\section{Anti-CD19-CAR T cell amplification in this study}

Proportions of anti-CD19-CAR T cells in peripheral blood was observed on $0,7,14,21,28$ and 60 days post CAR-T cell infusion. The median amplification peak of anti-CD19-CAR T cells in $\mathrm{CD}^{+}{ }^{+} \mathrm{T}$ cells was 14.1 (IQR 3.3-21.6)\% on 4 or 7 days after CAR-T cell infusion. The median amplification peak of antiCD19-CAR T cells was higher in patients with 3-4 grade of persistent cytopenia (24.1 (IQR 16.3-33.5)\%) than that of patients without such persistent cytopenia (8.2 (IQR 2.5-12.2)\%) ( $P=0.0006)$ (Figure 3d). Proportion of anti-CD19-CAR T cells could still be observed (more than $1 \%$ by FCM) at 21 days post CART cell infusion in 14 patients, while it could still be observed at 28 days post CAR-T cell infusion in 6 
patients. In our study, the result of patients whose CAR-T cells could be observed at 21 and 28 days after CAR-T cell infusion were associated with 3-4 grade of persistent cytopenia (Figure 3ef). All these $20 \mathrm{R} / \mathrm{R}$ DLBCL patients whose CAR-T cells could be observed at 21 and 28 days post CAR-T cell infusion were the patients who obtained CR or PR during this therapy.

\section{Follow up}

By the cutoff date, the median PFS and OS in all 38 patients was 14.32 (IQR 3-54) months and 15.11 (IQR 2-54) months. In patients with 3-4 grade of persistent time of hematological toxicity, the 1-year PFS and OS rates were $83.91 \%$ and $91.78 \%$ respectively. But in patients without such toxicity, the 1-year PFS and OS rates were $33.50 \%$ and $32.60 \%$ respectively. The PFS and OS were higher in patients with $3-4$ grade of persistent cytopenia than that of patients without such persistent cytopenia $\left(P_{P F S}=0.0019\right.$ and $\left.P_{O S}=0.0006\right)$ (Figure 4ab).

\section{Discussion}

Hematological toxicity is not an uncommon condition after anti-CD19-CAR T-cell therapy. Most $(90.4 \%)$ persistent cytopenia occurred between day 31 to 180 after CAR-T cell infusion in a study of toxicities in anti-CD19-CAR T-cell therapy [24]. In ZUMA-1 study [6, 12, 25], grade 3 or higher cytopenia is frequently in first 30 days after anti-CD19-CAR T-cell infusion and is associated with lymphodepleting chemotherapy with fludarabine and cyclophosphamide probably. It has been reported that the cytopenia could generally recover within several weeks even after six cycles of fludarabine and cyclophosphamide in chronic lymphocytic leukemia patients [26]. But in ZUMA-1 study, the cytopenia might persist for three months or later without evidence of marrow dysplasia or relapse [4]. Whether there is hematologic toxicity unrelated to the lymphodepleting chemotherapy?

The definition of prolongation and severity of cytopenia varies in diffenent studies [13, 27-29]. A high incidence of persistent CTCAE grade $\geq 3$ neutropenia (30-38\%), thrombocytopenia (21-29\%), and anemia (5-17\%) after day 21 has been reported. In our study, 14 in 38 R/R DLBCL patients developed 3-4 grade of persistent cytopenia after their anti-CD19-CAR T-cell therapy. To distinguish the persistent cytopenia from the hematological toxicity caused by lymphodepleting chemotherapy with fludarabine and cyclophosphamide, the persistent cytopenia in our study was defined as hematotoxicity lasting more than 56 days after CAR-T cell infusion. Meanwhile most AEs in addition to hematotoxicity had disappeared at 21-28 days after CAR-T cell infusion. The 3-4 grade of persistent cytopenia duration and recovery, and the clinical characteristics related to 3-4 grade of persistent cytopenia after anti-CD19-CAR T-cell therapy were analyzed in these 14 R/R DLBCL patients.

What's interesting in our study is that the trend curve of grade 3-4 neutropenia has two valleys in seven patients with persistent neutropenia at 7 days and 28-56 days after CAR-T infusion respectively. This 3-4 grade of persistent cytopenia was recovered at 8-18 weeks after CAR-T cell infusion with supportive therapy. The first valley of grade 3-4 neutropenia might be related to the lymphodepleting chemotherapy 
with fludarabine and cyclophosphamide. But what is the cause of the second valley of grade 3-4 neutropenia? Most of the infections after anti-CD19-CAR T-cell therapy occurred after their neutropenia had occurred in this study. Meanwhile, there was no evidence of severe marrow dysplasia or relapse in bone marrow at the second valley of grade 3-4 neutropenia in these patients. So the second valley of grade 3-4 neutropenia was not caused by the lymphodepleting chemotherapy, the infections and the marrow dysplasia or relapse, while it is most likely caused by the anti-CD19-CAR T-cell therapy. But the underlying mechanism is still unclear.

Other factors such as cytotoxic therapies, autologous or allogeneic HSCT have been hypothesized to cause the development of cytopenia $[10,28,30]$. In our study, other baseline characteristics of the R/R DLBCL patients did not differ between patients with and without 3-4 grade of persistent cytopenia, except that tumor load was larger in R/R DLBCL patients with persistent cytopenia before the anti-CD19-CAR Tcell therapy. Our results provide further evidence that anti-CD19-CAR T-cell therapy itself might lead to 3-4 grade of persistent cytopenia. A study reported that the increasing grade of CRS and ICANS, baseline cytopenias, CAR construct, higher peak C-reactive protein or ferritin levels were associated with persistent cytopenia for more than one month [18]. Another previous study demonstrated the same result that the grade $\geq 4$ CRS was associated with delayed hematopoietic recovery [30]. Anti-CD19-CAR T-cell induced cytolysis of CD19 positive lymphoma cells facilitates antigenic spreading, which might be associated with severe CRS [31-33]. Yet another study proved that the severe neutropenia until 60 days after antiCD19-CAR T-cell therapy was not associated with the incidence and severity of CRS, ICANS and the peak cytokine levels absolutely, while it was associated with hematopoietic reserve and baseline inflammation [23].

In our study, all R/R DLBCL patients who occured 3-4 grade of persistent cytopenia obtained ORR in their anti-CD19-CAR T-cell therapy. Furthermore, in all 26 patients who obtained ORR, the incidence of 3-4 grade of persistent cytopenia was higher in patients with high tumor load than that of patients without high tumor load. The other factors associated with 3-4 grade of persistent cytopenia include the peak level of IL-6, the grade of CRS, and the peak proportion of anti-CD19-CAR T cells in anti-CD19-CAR T-cell therapy. In addition, the persistent existence of CAR-T cells in peripheral blood at 21 and 28 days after infusion was associated with 3-4 grade of persistent cytopenia. Whereas, persistent cytopenia was not associated with grade of ICANS.

In a long-term follow-up study in patients after anti-CD19-CAR T-cell therapy, $16 \%$ of patients with persistent CR had sustained significant persistent cytopenia after infusion [30]. Similar results were obtained in our study. The PFS and OS were longer in patients with 3-4 grade of persistent cytopenia than that of patients without such persistent cytopenia.

\section{Conclusion}

In our study, 3-4 grade of persistent cytopenia occurred in 14 R/R DLBCL patients after anti-CD19-CAR Tcell therapy. All these 14 patients achieved ORR in this therapy. Our research proved that the 3-4 grade of 
persistent cytopenia was associated with high tumor load, higher peaks of IL- 6 and CAR T cells, higher grades of CRS, and longer PFS and OS. None of these patients developed severe infection or death as a direct result of infection in our study. Our study is helpful in predicting efficacy and severe hematologic side effects of anti-CD19-CAR T-cell therapy in R/R DLBCL patients.

\section{Abbreviations}

DLBCL: Diffuse large B-cell lymphoma, R/R: Relapsed/refractory, Chimeric antigen receptor T cell: CAR Tcell, AEs: Adverse events, CRS: Cytokine release syndrome, ICANS: Immune effector cell associated neurotoxic syndrome, ORR: Objective response rate, PFS: Progression free survival, OS: Overall survival, IL-6: Interleukin-6.

\section{Declarations}

\section{Acknowledgements}

We thank all our patients for their participation in our clinical trials.

We thank the Shanghai Genbase Biotechnology Co., Ltd. for providing us with anti-CD19-CAR-T-cells.

\section{Authors' contributions}

$X M L, M J L$ and QD contributed to the conception and design of the study and to the draft of the manuscript. XML and MJL were responsible for writing the manuscript. QD, XML, RC, CCL and JW were responsible to patients' enrollment and treatment, clinical data and management of patients. NM carried out statistical analysis. MJL and QL collected data and the follow-up of patients. All authors agreed to be accountable for all aspects of the work. All authors read and approved the final manuscript.

\section{Funding}

The National Natural Science Foundation of China (81900186).

Key R \& D projects of Ningxia Hui Autonomous Region囚2021BEG03036》

\section{Availability of data and materials}

The authors confirm that the data supporting the findings of this study are available within the article.

\section{Ethics approval and consent to participate}

This study was approved by the Medical Ethics Committee of the Tianjin First Center Hospital (Tianjin, China). (Approved No. of ethic committee: 2015002X and 2018N105KY). 
Informed consent was obtained from the participants. The patients agreed to participate in our Clinical trials. They agreed to the use of their data for our study. All the data andmaterial have been performed in accordance with the Declaration of Helsinki and conformed to relevant aspects of the ARRIVE guidelines.

This Clinical trials is registered at http://www.chictr.org.cn/index.aspx as ChiCTR-ONN-16009862 and http://www.chictr.org.cn/index.aspx as ChiCTR1800019622.

\section{Consent for publication}

Not applicable.

\section{Conflict of Interest}

The authors have no conflict of interest to report.

\section{Author details}

1 Department of Hematology, Tianjin First Central Hospital, School of Medicine, Nankai University, 24 Fukang Road, Tianjin, 300192, China.

2 Shanghai Genbase Biotechnology Co.,Ltd. 326 Edison Road, Shanghai, 201203, China.

\section{References}

1. Coiffier B, Lepage E, Briere J, Herbrecht R, Tilly H, Bouabdallah R, et al. CHOP chemotherapy plus rituximab compared with $\mathrm{CHOP}$ alone in elderly patients with diffuse large-B-cell lymphoma. $\mathrm{N}$ Engl $\mathrm{J}$ Med. 2002;346:235-42.

2. Flinn IW, van der Jagt R, Kahl BS, Wood P, Hawkins TE, Macdonald D, et al. Randomized trial of bendamustine-rituximab or R-CHOP/R-CVP in first-line treatment of indolent NHL or MCL: the BRIGHT study. Blood. 2014;123(19):2944-52.

3. Federico M, Luminari S, Dondi A, Tucci A, Vitolo U, Rigacci L, et al. R-CVP versus R-CHOP versus R-FM for the initial treatment of patients with advanced-stage follicular lymphoma: results of the FOLL05 trial conducted by the Fondazione Italiana Linfomi. J Clin Oncol. 2013;31(12):1506-13.

4. Locke FL, Ghobadi A, Jacobson CA, et al. Long-term safety and activity of axicabtagene ciloleucel in refractory large B-cell lymphoma (ZUMA-1): a single-arm, multicentre, phase 1-2 trial. Lancet Oncology. 2019;20(1):31-42.

5. Chow VA, Shadman M, Gopal AK. Translating anti-CD19 CAR T-cell therapy into clinical practice for relapsed/refractory diffuse large B-cell lymphoma. Blood. 2018;132(8):777-81.

6. Schuster SJ, Bishop MR, Tam CS, et al. Tisagenlecleucel in Adult Relapsed or Refractory Diffuse Large B-Cell Lymphoma. N Engl J Med. 2019;380(1):45-56.

7. Neelapu SS, Locke FL, Bartlett NL, et al. Axicabtagene Ciloleucel CAR T-Cell Therapy in Refractory Large B-Cell Lymphoma. N Engl J Med. 2017;377(26):2531-44. 
8. Karschnia P, Jordan JT, Forst DA, et al. Clinical presentation, management, and biomarkers of neurotoxicity after adoptive immunotherapy with CAR T cells. Blood. 2019;133(20):2212-21.

9. Shimabukuro-Vornhagen A, Godel P, Subklewe M, et al. Cytokine release syndrome. J Immunother Cancer. 2018;6(1):56.

10. Hay KA, Hanafi LA, Li D, et al. Kinetics and biomarkers of severe cytokine release syndrome after CD19 chimeric antigen receptor-modified T-cell therapy. Blood. 2017;130(21):2295-306.

11. Neelapu SS, Tummala S, Kebriaei P, et al. Chimeric antigen receptor T-cell therapy-assessment and management of toxicities. Nat Rev Clin Oncol. 2018;15(1):47-62.

12. Hill JA, Li D, Hay KA, et al. Infectious complications of CD19-targeted chimeric antigen receptormodified T-cell immunotherapy. Blood. 2018;131(1):121-30.

13. George RN, Krishna VK, Denise P, et al. Incidence and risk factors associated with a syndrome of persistent cytopenias after CAR-T cell therapy (PCTT). Leuk Lymphoma. 2020;61(4):940-3.

14. Kochenderfer JN, Somerville RPT, Lu T, et al. Long-duration complete remissions of diffuse large B cell lymphoma after anti-CD19 chimeric antigen receptor T cell therapy. Mol Ther. 2017;25(10):224553.

15. Brudno JN, Kochenderfer JN. Toxicities of chimeric antigen receptor T cells: recognition and management. Blood. 2016;127(26):3321-30.

16. Maude SL, Laetsch TW, Buechner J, et al. Tisagenlecleucel in children and young adults with B-cell lymphoblastic leukemia. N Engl J Med. 2018;378(5):439-48.

17. Kai R, Ariel P, Pierre S, et al. CAR-HEMATOTOX: a model for CAR T-cell-related hematologic toxicity in relapsed/refractory large B-cell lymphoma. Blood. 2021;138(24):2499-513.

18. Jain $T$, Knezevic A, Pennisi $M$, et al. Hematopoietic recovery in patients receiving chimeric antigen receptor T-cell therapy for hematologic malignancies. Blood Adv. 2020;4(15):3776-87.

19. Cheson BD, Fisher RI, Barrington SF, Cavalli F, Schwartz LH, Zucca E, et al. Recommendations for initial evaluation, staging, and response assessment of Hodgkin and non-Hodgkin lymphoma: the Lugano classification. J Clin Oncol. 2014;32(27):3059-68.

20. Lee DW, Gardner R, Porter DL, Louis CU, Ahmed N, Jensen M, et al. Current concepts in the diagnosis and management of cytokine release syndrome. Blood. 2014;124(2):188-95.

21. Lee DW, Santomasso BD, Locke FL, Ghobadi A, Turtle CJ, Brudno JN, et al. ASTCT Consensus Grading for Cytokine Release Syndrome and Neurologic Toxicity Associated with Immune Effector Cells. Biol Blood Marrow Transplant. 2019;25(4):625-38.

22. Taplitz RA, Kennedy EB, Bow EJ, et al. Antimicrobial Prophylaxis for Adult Patients With CancerRelated Immunosuppression: ASCO and IDSA Clinical Practice Guideline Update. J Clin Oncol. 2018;36(30):3043-54.

23. Kai Rejewski A, Perez P, Sesques, et al. CAR-HEMATOTOX: a model for CAR T-cell-related hematologic toxicity in relapsed/refractory large B-cell lymphoma. Blood. 2021;138(24):2499-513. 
24. Wudhikarn K, Pennisi M, Garcia-Recio M, et al. DLBCL patients treated with CD19 CAR T cells experience a high burden of organ toxicities but low nonrelapse mortality. Blood Adv. 2020;4(13):3024-33.

25. Jain MD, Davila ML. Concise review: emerging principles from the clinical application of chimeric antigen receptor T cell therapies for B cell malignancies. Stem Cells. 2018;36(1):36-44.

26. Joffe E, Ariela Arad N, Bairey O, et al. Persistently low lymphocyte counts after FCR therapy for chronic lymphocytic leukemia are associated with longer overall survival. Hematol Oncol. 2018;36(1):128-35.

27. Logue JM, Zucchetti E, Bachmeier CA, et al. Immune reconstitution and associated infections following axicabtagene ciloleucel in relapsed or refractory large B-cell lymphoma. Haematologica. 2020;106(4):978-86.

28. Fried S, Avigdor A, Bielorai B, et al. Early and late hematologic toxicity following CD19 CAR-T cells. Bone Marrow Transplant. 2019;54(10):1643-50.

29. Sesques P, Ferrant E, Safar V, et al. Commercial anti-CD19 CAR T cell therapy for patients with relapsed/refractory aggressive B cell lymphoma in a European center. Am J Hematol. 2020;95(11):1324-33.

30. Cordeiro A, Bezerra ED, Hirayama AV, et al. Late events after treatment with CD19-targeted chimeric antigen receptor modified T cells. Biol Blood Marrow Transplant. 2020;26(1):26-33.

31. Brossart P. The Role of Antigen Spreading in the Efficacy of Immunotherapies. Clin Cancer Res. 2020;26(17):4442-7.

32. Gulley JL, Madan RA, Pachynski R, et al. Role of Antigen Spread and Distinctive Characteristics of Immunotherapy in Cancer Treatment. J Natl Cancer Inst. 2017;109(4).

33. Faramand R, Jain M, Staedtke $V$, et al. Tumor Microenvironment Composition and Severe Cytokine Release Syndrome (CRS) Influence Toxicity in Patients with Large B-Cell Lymphoma Treated with Axicabtagene Ciloleucel. Clin Cancer Res. 2020;26(18):4823-31.

\section{Tables}

Table 1. Patients baseline characteristics 


\begin{tabular}{|c|c|c|c|c|c|c|c|}
\hline & Sex & Age & Subtype & Stage & IPI & Prelines of therapy & Poor prognosis \\
\hline P1 & $M$ & 56 & Non-GCB & III & 3 & 3 & C-MYC BCL6 (DE),TP53 \\
\hline P2 & M & 25 & GCB & IV & 2 & 3 & No \\
\hline P3 & M & 33 & Non-GCB & III & 2 & 3 & C-MYC BCL6 (DH) \\
\hline P4 & M & 45 & GCB & IV & 3 & 4 & C-MYC BCL6 (DE) \\
\hline P5 & M & 72 & Non-GCB & II & 2 & 2 & No \\
\hline P6 & M & 49 & GCB & III & 2 & 4 & C-MYC BCL6 (DE),TP53 \\
\hline P7 & $\mathrm{F}$ & 79 & GCB & III & 2 & 3 & C-MYC BCL2 (DE) \\
\hline P8 & M & 55 & GCB & IV & 3 & 3 & C-MYC BCL2 BCL6 (TH) \\
\hline P9 & $M$ & 64 & Non-GCB & IV & 3 & 4 & No \\
\hline P10 & $\mathrm{F}$ & 29 & GCB & III & 3 & 4 & C-MYC BCL2 (DE) \\
\hline P11 & $\mathrm{F}$ & 33 & Non-GCB & III & 2 & 3 & No \\
\hline P12 & M & 40 & Non-GCB & IV & 3 & 2 & C-MYC BCL6 (DT) \\
\hline P13 & $M$ & 46 & Non-GCB & III & 3 & 4 & C-MYC BCL6 (DE) \\
\hline P14 & M & 22 & GCB & IV & 3 & 5 & No \\
\hline P15 & M & 35 & Non-GCB & II & 3 & 2 & C-MYC BCL6 (DH) ,TP53 \\
\hline P16 & M & 41 & Non-GCB & III & 2 & 2 & C-MYC BCL2 (DE) \\
\hline P17 & $M$ & 46 & GCB & III & 2 & 4 & No \\
\hline P18 & $M$ & 56 & Non-GCB & IV & 3 & 3 & C-MYC BCL2 (DH) ,TP53 \\
\hline P19 & M & 38 & GCB & IV & 2 & 3 & No \\
\hline P20 & $\mathrm{F}$ & 72 & Non-GCB & III & 3 & 3 & TP53 \\
\hline P21 & $M$ & 50 & Non-GCB & IV & 4 & 3 & C-MYC BCL6 (DE) \\
\hline P22 & $M$ & 41 & Non-GCB & III & 3 & 5 & TP53 \\
\hline P23 & $\mathrm{F}$ & 55 & GCB & IV & 2 & 4 & No \\
\hline P24 & $\mathrm{F}$ & 35 & GCB & II & 2 & 3 & No \\
\hline P25 & $M$ & 62 & Non-GCB & III & 3 & 4 & No \\
\hline P26 & $\mathrm{F}$ & 52 & Non-GCB & III & 3 & 5 & C-MYC BCL2 (DH) \\
\hline P27 & $\mathrm{F}$ & 70 & Non-GCB & IV & 2 & 3 & No \\
\hline P28 & M & 58 & GCB & IV & 2 & 4 & C-MYC BCL6 (DE) \\
\hline
\end{tabular}




\begin{tabular}{|llllllll|} 
P29 & F & 71 & GCB & III & 3 & 3 & C-MYC BCL2 BCL6 (TH) \\
P30 & M & 76 & Non-GCB & IV & 3 & 3 & C-MYC BCL2 (DE) \\
P31 & M & 51 & Non-GCB & III & 3 & 4 & C-MYC BCL6 (DE),TP53 \\
P32 & F & 66 & GCB & III & 3 & 3 & No \\
P33 & M & 52 & Non-GCB & II & 2 & 4 & C-MYC BCL2 BCL6 (TH),TP53 \\
P34 & M & 45 & GCB & III & 2 & 3 & C-MYC BCL6 (DT) \\
P35 & F & 72 & GCB & IV & 2 & 5 & C-MYC BCL2 (DE) \\
P36 & M & 61 & GCB & IV & 3 & 3 & TP53 \\
P37 & M & 57 & Non-GCB & III & 2 & 5 & C-MYC BCL2 (DH) ,TP53 \\
P38 & M & 79 & Non-GCB & III & 4 & 3 & No \\
\hline
\end{tabular}

NOTE: GCB:Germinal center B-cell-like lymphoma, Non-GCB:Non-Germinal center B-cell-like lymphoma,DH:Double Hit, TH:Triple Hit, DE:Double expression, TE: Triple expression, TP53:TP53 is mutation or deletion

Table 2. Comparison of baseline characteristics between the two groups 


\begin{tabular}{|c|c|c|c|}
\hline & $\begin{array}{l}\text { With persistent cytopenia } \\
(n=14)\end{array}$ & $\begin{array}{l}\text { Without persistent cytopenia } \\
(n=24)\end{array}$ & $P$ value \\
\hline \multicolumn{4}{|l|}{ Sex } \\
\hline Male:Femal & $9: 5$ & $16: 8$ & 1.000 \\
\hline Age (years) & $50.6 \pm 17.8$ & $53.4 \pm 13.8$ & 0.595 \\
\hline \multicolumn{4}{|l|}{ Subtype } \\
\hline Non-GCB: GCB & $6: 8$ & 15:9 & 0.317 \\
\hline \multicolumn{4}{|l|}{ Stage } \\
\hline I-IIIII-IV & $0: 14$ & $4: 20$ & 0.275 \\
\hline \multicolumn{4}{|l|}{ IPI(Score) } \\
\hline $7-2: 3-4$ & $5: 9$ & $12: 12$ & 0.505 \\
\hline \multicolumn{4}{|l|}{ Poor prognosis } \\
\hline With $\square$ Without & $8: 6$ & $17: 7$ & 0.486 \\
\hline $\begin{array}{l}\text { Max tumor } \\
\text { diameter }(\mathrm{cm})\end{array}$ & $12.89 \pm 4.91$ & $5.04 \pm 1.88$ & 0.001 \\
\hline Prelines of therapy & $2.78 \pm 0.69$ & $2.50 \pm 0.51$ & 0.337 \\
\hline
\end{tabular}

NOTE:Poor prognosis including:DH:Double Hit, TH:Triple Hit, DE:Double expression, TP53:TP53 is mutation or deletion

Table 3. AEs in the process of anti-CD19 CAR-T cell therapy 


\section{With persistent cytopenia}

\n=14ロ
Without persistent cytopenia

[n=24!

\section{Events}

$P$ value

\section{General condition}

Temperature $\geq 38^{\circ} \mathrm{C}$ (fever)

Chills

Rash

Muscular weakness

Hypotension

Hypoxia

Fatigue

Organ toxicities

Gastrointestinal

Nausea

Vomiting

Decreased appetite

Diarrhea

Abdominal pain

\section{Cardiac}

Tachycardia

Arrhythmias

Respiratory

Dyspnoea

Cough

Pleural effusion

Renal

Increased creatinine

Oliguria

Hepatic
$13 / 14$ (92.86\%)

5/14 (35.71\%)

$3 / 14(21.43 \%)$

$5 / 14(35.71 \%)$

$2 / 14$ (14.29\%)

2/14 (14.29\%)

$11 / 14(78.57 \%)$

$4 / 14(28.57 \%)$

$3 / 14(21.43 \%)$

$7 / 14(50.00 \%)$

$4 / 14(28.57 \%)$

6/14 (42.86\%)
20/24 (83.33\%)

9/24 (37.50\%)

$3 / 24$ (12.50\%)

8/24 (33.33\%)

$0 / 24(0.00 \%)$

$0 / 24(0.00 \%)$

$15 / 24$ ( $62.50 \%)$

0.472

1.000

0.65

1.000

0.129

0.129
0.663
$3 / 24(33.33 \%) \quad 0.387$

$1 / 24(4.17 \%) \quad 0.132$

$4 / 24(16.67 \%) \quad 0.061$

$1 / 24(4.17 \%) \quad 0.052$

$5 / 24(20.83 \%)$

0.266
$3 / 14(21.43 \%) \quad 2 / 24(8.33 \%)$

$0 / 24(0.00 \%)$

0.337

$1 / 14(7.14 \%)$

0.368
$2 / 14(14.29 \%)$

$4 / 14(28.57 \%)$

$5 / 14(35.71 \%)$

$3 / 14(21.43 \%)$

$5 / 14(35.71 \%)$

$4 / 14(28.57 \%)$

$2 / 24(8.33 \%)$
0.129

$5 / 24(20.83 \%) \quad 0.699$

$6 / 24(25.00 \%)$

0.712 
Increased serum bilirubin levels

2/14 (14.29\%)

$3 / 24(12.50 \%)$

1.000

Neurological

Headache

$6 / 14(42.86 \%)$

9/24 (37.50\%)

1.000

Dizziness

2/14 (14.29\%)

$0 / 24(0.00 \%)$

0.129

Confused

$1 / 14(7.14 \%)$

0/24 (0.00\%)

0.368

Somnolence

2/14 (14.29\%)

$2 / 24(8.33 \%)$

0.616

Aphasia

$1 / 14(7.14 \%)$

$0 / 24(0.00 \%)$

0.368

Disorientation

$1 / 14(7.14 \%)$

$0 / 24(0.00 \%)$

0.368

\section{Hematological}

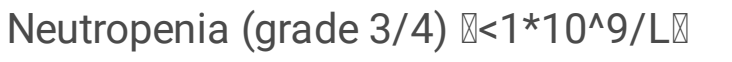

$10 / 14(71.43 \%)$

$3 / 24(12.50 \%)$

0.000

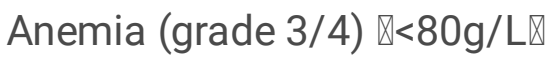

$7 / 14(50.00 \%)$

$2 / 24(8.33 \%)$

0.006

Thrombocytopenia (grade 3/4) $\left(<50 * 10^{\wedge} 9 / \mathrm{L}\right)$

$9 / 14(64.29 \%)$

$2 / 24(8.33 \%)$

0.000

NOTE: The AEs are according to the National Cancer Institute's Common Terminology

Criteria for Adverse Events (version 4.03).

Figures 
a

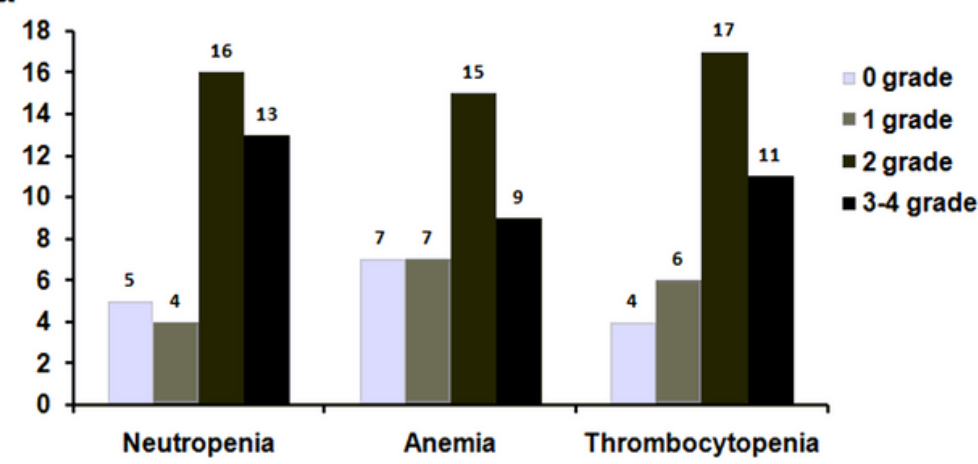

b

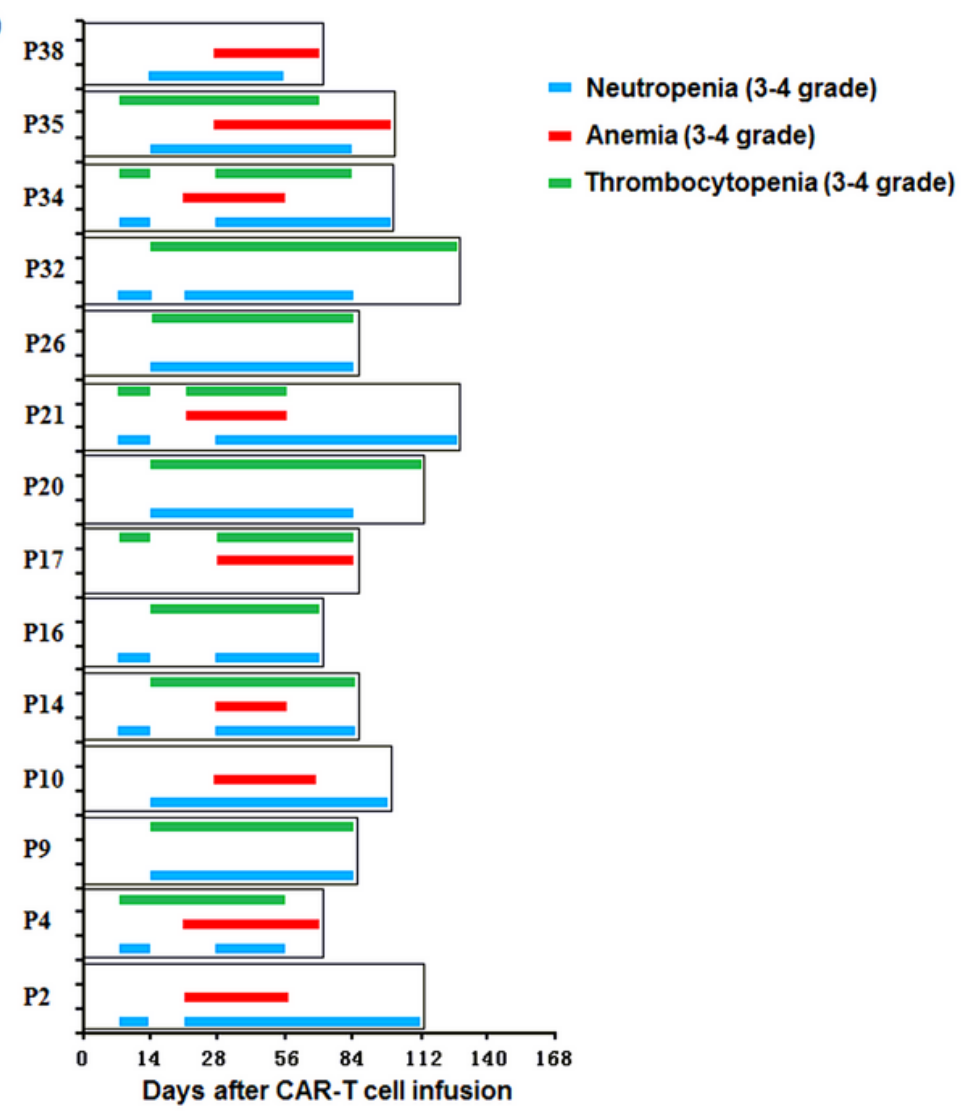

C

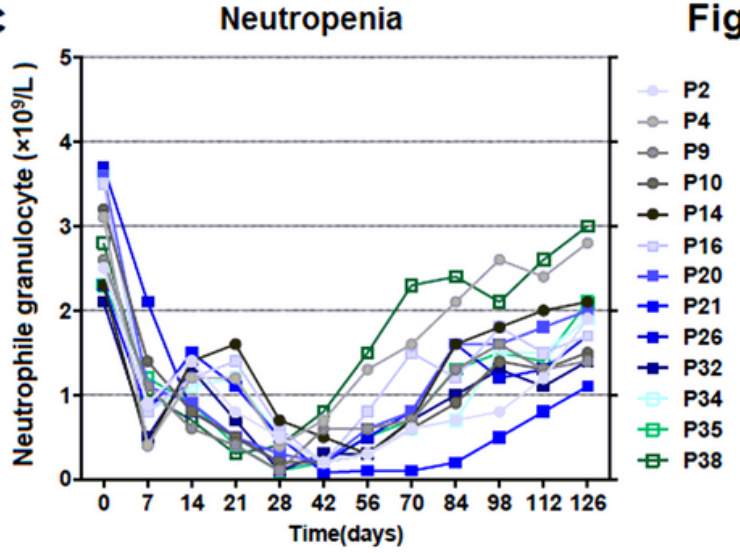

d

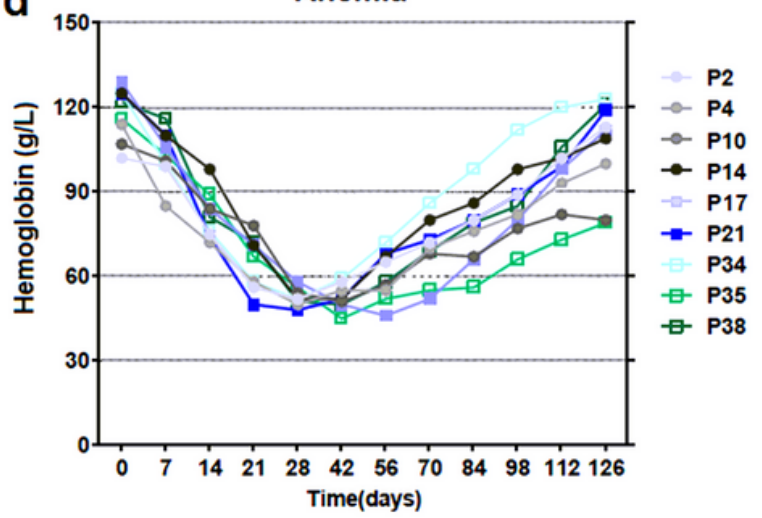

E

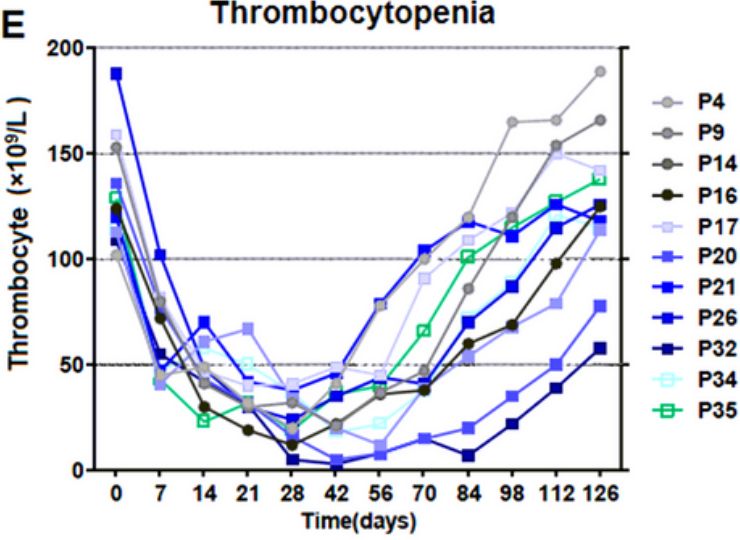

Figure 1

Hematological toxicity of anti-CD19-CAR T-cell therapy. a. The neutropenia, anemia and thrombocytopenia was diagnosed with 0-4 grade after anti-CD19-CAR T-cell therapy. b. The different types and persistent time of cytopenia in the 14 patients who were defined as 3-4 grade of persistent cytopenia. ce. The trend of blood cell changes in patients with 3-4 grade of neutropenia, anemia and thrombocytopenia. 

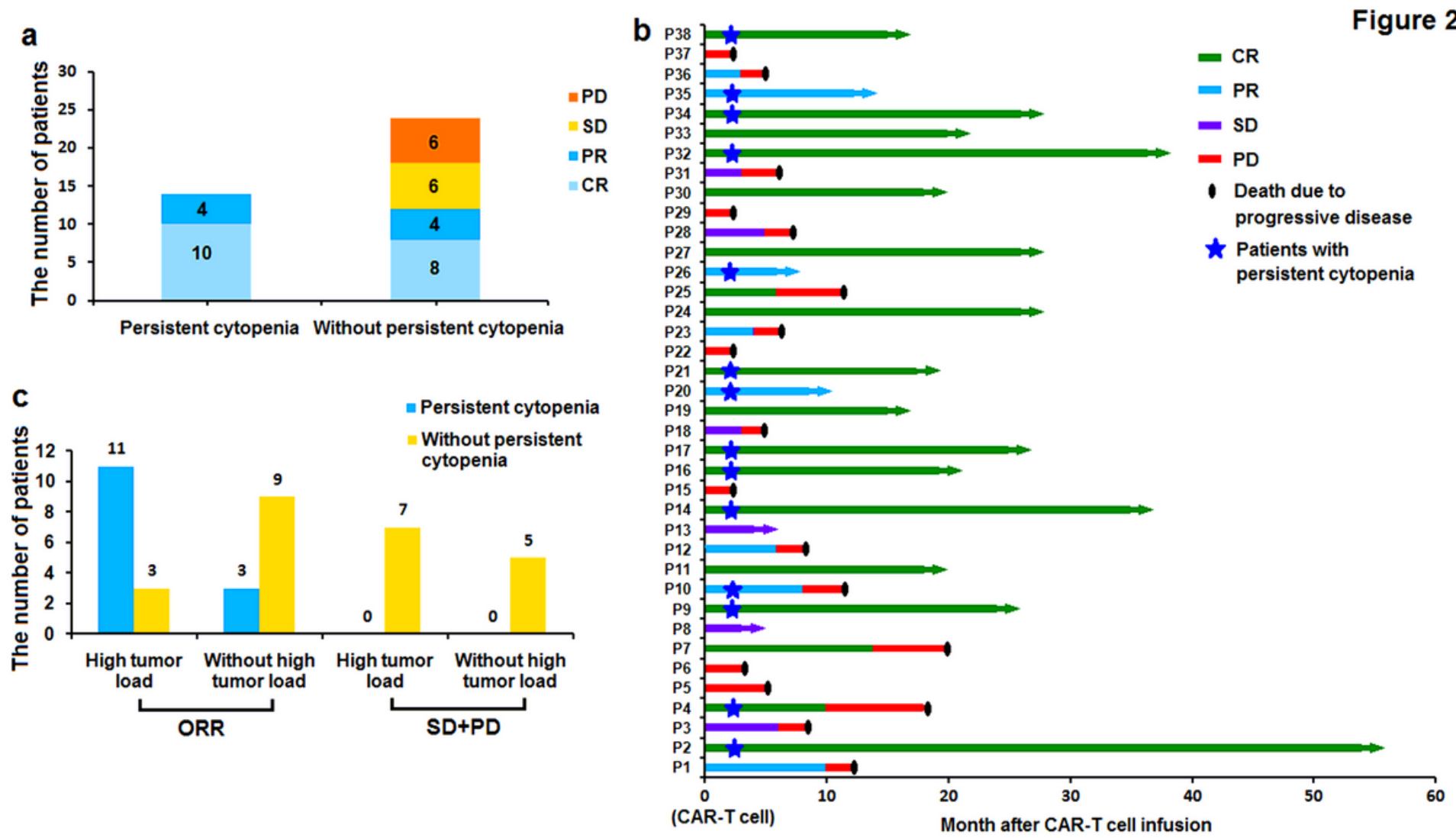

Figure 2

a. All the 14 patients with 3-4 grade of persistent cytopenia obtained CR and PR in our study, while no patient who obtained SD and PD had such cytopenia in CAR T-cell therapy. $\mathbf{b}$. The clinical response to anti-CD19-CAR T-cell therapy, disease progression and survival time of all the 38 patients. c. The incidence of 3-4 grade of persistent cytopenia was higher in patients with high tumor load in 26 patients obtained ORR. 

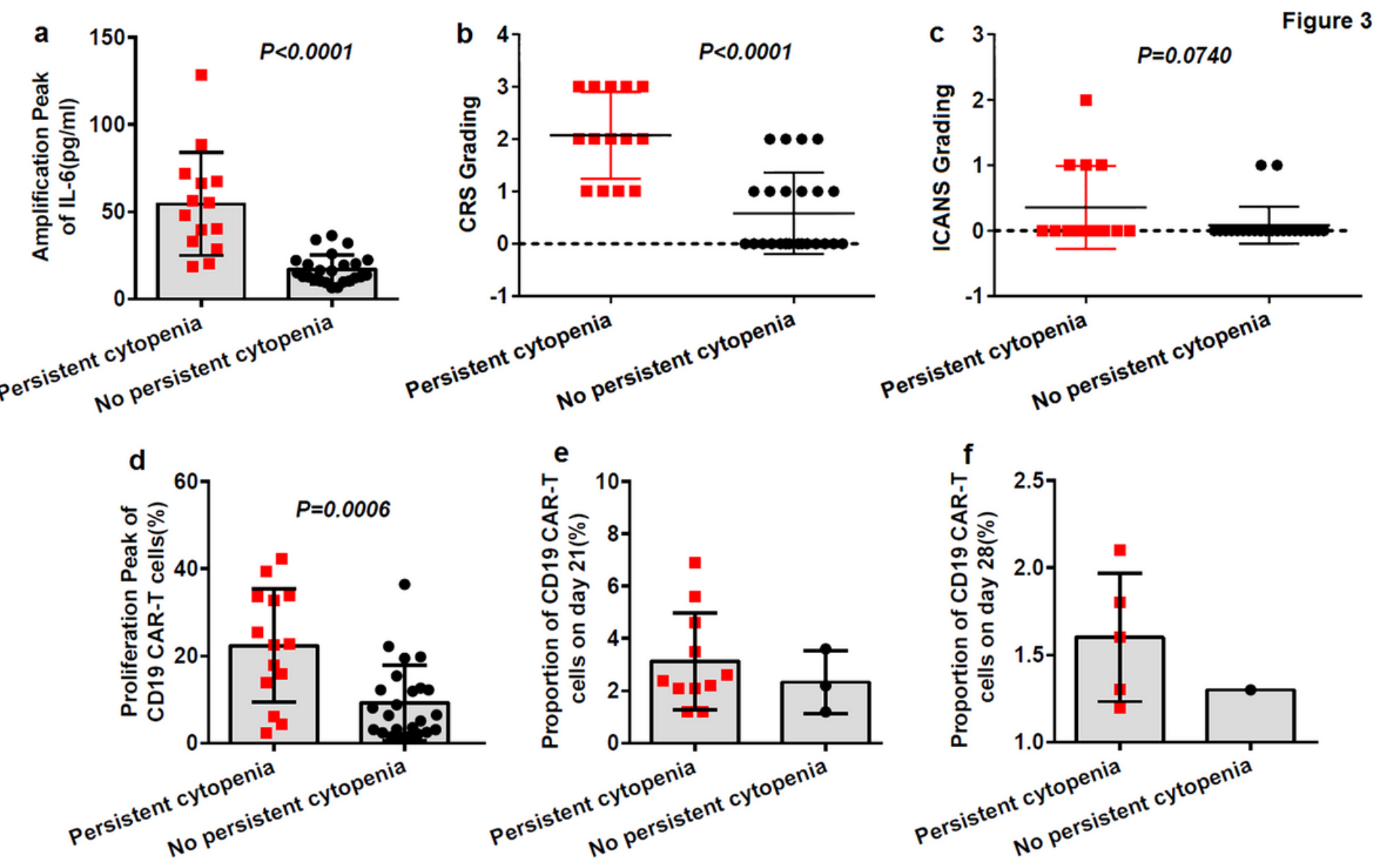

Figure 3

AEs of anti-CD19-CAR T-cell therapy. a. The mean of peak of IL- 6 in patients with 3-4 grade of persistent cytopenia was more higher. $\mathbf{b}$. The higher grades of CRS were higher in patients with 3-4 grade of persistent cytopenia than that of in patients without such persistent cytopenia. $\mathbf{c}$. There was no difference of 3-4 grade of persistent cytopenia between different grades of ICANS. $\mathbf{d}$. The median amplification peak of CAR T cells was higher in patients with 3-4 grade of persistent cytopenia than that of in patients without such persistent cytopenia. $\mathbf{e}$ - . The majority of patients whose CAR-T cells could be observed at 21 and 28 days after CAR-T cell infusion were associated with 3-4 grade of persistent cytopenia. 

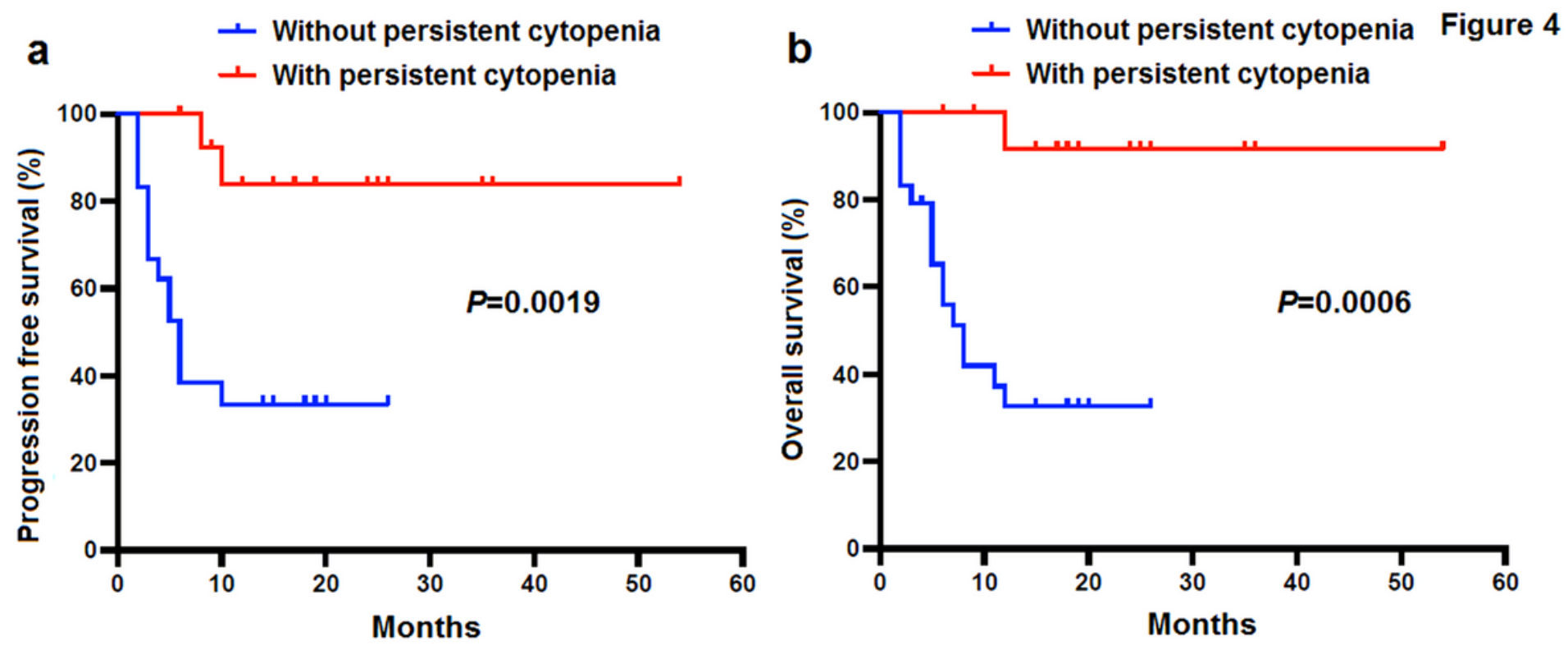

Figure 4

Follow up after anti-CD19-CAR T-cell therapy. a-b. The PFS and OS were more higher in patients with 3-4 grade of persistent cytopenia. 